\title{
Von Willebrand factor in follicular fluid in women undergoing IVF/ICSI: A "ghost" associated with oocyte and embryo quality
}

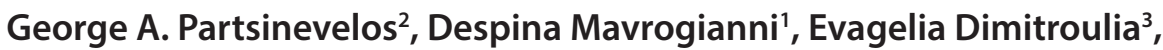 \\ Vasiliki Dinopoulou' ${ }^{1}$, Dimitris Loutradis ${ }^{4}$, Peter Drakakis ${ }^{1}$
}

\author{
'Division of Human Reproduction, IVF Unit, 1st Department of Obstetrics and Gynaecology, Alexandra Hospital, \\ National and Kapodistrian University of Athens, Medical School, Athens, Greece \\ 2Institute of Life IASO, IASO Maternity Hospital, Marousi, Athens, Greece \\ ${ }^{3}$ Microbiology Department, National and Kapodistrian University of Athens, Medical School of Athens, Athens, \\ Greece \\ ${ }^{4}$ Institute Gonimotitas, National and Kapodistrian University of Athens, Medical School, Athens, Greece
}

\section{Corresponding Author}

George A. Partsinevelos, MD, PhD, Consultant Obstetricial-Gynaecologist, Subspecialist in Reproductive Medicine and Minimal Access Surgery, Institute of Life IASO, IASO Maternity Hospital, 37-39, Kifissias Avenue, 15123 Maroussi, Athens, Greece e-mail: partsiobgyn@yahoo.com

\begin{abstract}
Introduction: The purpose of the present study to determine the presence of Von Willebrand factor (vWF) in the follicular fluid of women undergoing IVF/ICSI and to investigate the association of its concentration with oocyte and embryo quality.

Methods: We conducted a prospective observational study based in women undergoing in vitro fertilization treatment. vWF concentrations in follicular fluid samples collected during transvaginal oocyte retrieval were measured using an enzyme-linked immunosorbent assay (Elisa). They were also analyzed by the VIDAS ${ }^{\circledR} \mathrm{VWF}$ method for confirmation of the results. The latter is an automated quantitative test for the measurement of vWF in human plasma, using the ELFA technique (enzyme-linked fluorescent assay). $\mathrm{vWF}$ concentrations were correlated to oocyte and embryo quality.

Results: Contrary to previous reports, vWF was determined in follicular fluid. A higher percentage of good quality oocytes were found in the presence of lower vWF follicular fluid levels. Similarly, better quality embryos were derived from oocytes retrieved from follicles with lower vWF follicular fluid levels.

Conclusions: Contrary to existing evidence to date, vWF was found in the follicular fluid of women undergoing IVF/ICSI treatment. In addition, the quality of oocytes and embryos was adversely related to vWF follicular fluid levels. In fact, a better outcome of IVF/ICSI treatment was suggested when the levels of vWF in follicular fluid were low. In this context, vWF follicular fluid levels could be used as a prognostic factor for oocyte and embryo quality.
\end{abstract}

Key Words: von Willebrand Factor, IVF, ICSI, oocyte quality, embryo quality 


\section{Introduction}

Ovarian follicle is one of the most rapidly remodeling structures in the body, undergoing a perpetual cycle of events characterized by cellular proliferation, migration and diverse functional activity. Although plenty of studies have looked at hormonal and growth factors involved folliculogenesis, the role of other factors, such as coagulation proteins is still poorly defined $^{1}$. In several aspects, the composition of follicular fluid resembles that of plasma. In fact, the electrolyte profile and albumin content are similar ${ }^{2-5}$. In previous studies analyzing follicular fluid, several components of the haemostatic system that are present in the blood circulation such as prothrombin and components of the prothrombinase enzyme complex, including factors V, VII and X, were also found to be present in the follicular fluid ${ }^{1}$. Furthermore, a role of thrombin in folliculogenesis through its hormone-like activity via receptor mediated intracellular signaling was also hinted.

Von Willebrand factor (vWF) is a large, multimeric glycoprotein implicated in hemostasis. It is synthesized and stored in vascular endothelial cells and megakaryocytes and is released in the blood stream mainly from endothelial cells upon stimulation. Furthermore, vWF is also found in subendothelial connective tissue. vWF has a key role in hemostasis as it mediates the adhesion of platelets to subendothelial connective tissue and it acts as a carrier binding blood clotting factor VIII $^{6}$. vWF is partitioned between two pathways in the Golgi of endothelial cells. The majority, up to $95 \%$, is straightly secreted mainly into the blood stream, whereas the remainder is stored in cytoplasmic granules specific for endothelium, known as Weibel Palade bodies (WPB) ${ }^{7,8}$. Its presence in the follicular fluid has never been reported to date. In fact, a previous study concluded that $v W F$ is absent in the follicular fluid ${ }^{1}$. However, on a theoretical basis, the presence of vWF could be anticipated, given that this factor is expressed in the endothelial cells and has been associated with morphological changes of microvasculature in atretic follicles ${ }^{9}$.

The present study was undertaken to determine whether vWF is present in the follicular fluid of women undergoing IVF/ICSI and to determine if a correlation between vWF follicular fluid levels and oocyte maturity as well as quality of the embryos developed can be established.

\section{Materials and Methods}

\section{Materials}

Twenty-five women undergoing IVF/ICSI treatment for several indications (unexplained infertility, tubal disease, male factor infertility and anovulation) at the Division of Human Reproduction, IVF Unit of the $1^{\text {st }}$ Department of Obstetrics and Gynaecology, National and Kapodestrian University of Athens, School of Medicine in Alexandra Hospital, were enrolled into the study.

The inclusion criteria were premenopausal woman, 25-40 years of age and a normal hormone profile (according to WHO guidelines), who had not received ovulation induction or any other hormonal treatment for at least three months before entering the study. Approval from the Ethics Committee of our Institute was obtained before the study began and a written informed consent was provided by all participants, according to the Helsinki Declaration of ethical principles for medical research involving human subjects.

\section{Methods}

\section{Stimulation Protocol}

Ovarian stimulation was achieved either by long luteal GnRH-agonist protocol or by short GnRHagonist protocol.

The long protocol was briefly as follows: On day 21 of the previous cycle, a baseline ultrasound scan 
was performed and Buserelin (Suprefact, Hoechst, Germany) intranasal spray was commenced at a dose of $100 \mu \mathrm{g} 5$ times daily (every 4 hours, omitting the 4 A.M. dose). The down regulation period before rFSH administration was 15-24 days. The extent of ovarian suppression was evaluated by ultrasound scan and serum $\mathrm{E}_{2}$ levels $(\leq 40 \mathrm{pg} / \mathrm{ml})$ before starting exogenous gonadotropin administration. Stimulation was conducted with $\mathrm{rFSH}$ (Puregon, Organon, The Netherlands) at a fixed dose of 200 IU daily for the first 6 days. Ultrasound scan along with serum estradiol levels determination was performed on day 7 and every day onwards until the day after hCG (Pregnyl, Organon, The Netherlands) administration and the dose of rFSH was adjusted according to ovarian response from day 7 of gonadotropin administration. GnRH-agonist administration was continued to the day of hCG administration for triggering final oocyte maturation. HCG 10.000 IU was given intramuscularly when the mean diameter of at least two leading follicles was $\geq 18 \mathrm{~mm}$ and serum $\mathrm{E}_{2}$ was rising. The interval between the last gonadotropin injection and hCG was no more than 24 hours. Thirty five to 36 hours after hCG, oocyte retrieval was performed by transvaginal echo-guided ovarian puncture. The luteal phase was supported with 2,500 IU of hCG injected on days of oocyteretrieval, embryo transfer (day 2 after the ovum retrieval) and day 6 after oocyte retieval ${ }^{10}$.

The short protocol was briefly as follows: Patients started Buserelin (Suprefact, Hoechst, Germany) intranasal spray at a dose of $100 \mu \mathrm{g} 5$ times daily (every 4 hours, omitting the 4 A.M. dose) on cycle day 2 . Administration of gonadotropins started on day 3 at a fixed dose of $200 \mathrm{IU}$ rFSH (Puregon, Organon, The Netherlands). Thenceforth, the procedure was the same as described previously in the long protocol ${ }^{11}$.

It should be noted that a single clinician performed all ultrasound scans as well as all oocyte retriev- als and embryo-transfers. Furthermore, the same embrylogist assessed oocytes and embryos and performed fertilization with IVF/ICSI for all patients.

Upon stripping of the cumulus and assessment of nuclear and cytoplasmic maturity, oocytes were graded on a 5-level scale from 1 (the best quality) to 5 (the poor quality) under a light microscope on the day of oocyte retrieval, whereas the embryos were graded according to their morphologic appearance on a 4-level scale from $3+$ (the best quality) to 3 , $2+, 2$ (the poor quality) under a light microscope on the day of transfer according to the standard practice of the Embryology team of the Division of Human Reproduction, IVF Unit, $1^{\text {st }}$ Department of Obstetrics and Gynaecology, National and Kapodistrian University of Athens, School of Medicine in Alexandra Hospital.

After transvaginal echo-guided ovarian puncture with a $16 \mathrm{G}$ needle, the fluid aspirated from each follicle was collected. Only clear, non-blood contaminated follicular fluid was used for the study. The samples of follicular fluid were centrifuged at 400 $\mathrm{g}$ for 6 minutes, and the supernatants were stored at $-23{ }^{\circ} \mathrm{C}$ for subsequent analysis.

\section{Determination of Von Willebrand Factor}

Von Willebrand factor concentrations in follicular fluid samples were measured using an enzymelinked immunosorbent assay (IMUBIND ${ }^{\circledR}$ VFW ELISA kit; American diagnostica inc., Stamford, USA). The vWF ELISA kit that was used is an enzyme-linked immunoassay for the quantitation of vWF antigen in human plasma. This assay is for research use only. The IMUBIND VFW ELISA is a "sandwich" ELISA using a goat antibody as the capture antibody. Samples incubate in precoated micro-test wells and the same polyclonal antibody, horseradish peroxidase (HRP) conjugated, is used to detect the bound vWF antigen. The addition of perborate $/ 3,3^{\prime}$, 5,5' 'tetramethylbenzidine (TBM) 
substrate, and its subsequent reaction with the HRP, creates a blue coloured solution. Sensitivity is enhanced by the addition of a $0.5 \mathrm{M}$ sulfuric acid stop solution, yielding a yellow coloured solution. VWF levels are determined by measuring and comparing the absorbance of sample solutions at $450 \mathrm{~nm}$ (Photometer: DUNEX MRX) against those of a standard curve developed using calibrated antigen. Samples of follicular fluid were diluted 1:10 in sample Buffer (prepared by adding BSA to Wash Buffer to a concentration of 3\% W/V-3gm BSA $100 \mathrm{~mL}$ Wash Buffer) and analyzed in duplicate. The detection limit was theoretically $0.25 \mathrm{mU} / \mathrm{mL}$ and analytically around $0.08 \pm 0.03 \mathrm{mU} / \mathrm{mL}$. For the calculation of results the mean absorbance value for each diluted sample was used to interpolate each vWF concentration from the standard curve. The concentration determined from the standard curve was multiplied by 10 (the dilution factor) to obtain the vWF concentration in the original follicular fluid sample.

The standard curve was constructed by plotting the mean absorbance value calculated for each $v W F$ standard versus its corresponding concentration. A standard curve was generated each time the assay was performed.

The follicular fluid samples were also analyzed by the VIDAS $₫$ vWF method for confirmation of the results. The VIDAS $®$ vWF method is an automated quantitative test for use on the VIDAS instruments, for the measurement of vWF in human plasma, using the ELFA technique (enzyme-linked fluorescent assay). The assay principle combines a two-step enzyme immunoassay sandwich method with a final fluorescent detection (ELFA). The Solid Phase Receptacle (SPR) serves as the solid phase as well as the pipetting device for the assay. Reagents for the assay are ready to use and predispensed in the sealed reagent strips. All of the assay steps are performed automatically by the instrument. The reaction medium is cycled in and out of the SPR several times. First step: the sample is collected then transferred into the well containing diluent. The diluted sample is cycled in and out of the SPR several times to enable the antigen to bind with the immunoglobulins absorbed on the inner side of the SPR. Unbound components are eliminated during the washing phase. Second step: the conjugate is cycled in and out of the SPR and binds with the antigen retained during the first step. Unbound components are eliminated during the washing steps.

During the final detection step, the substrate (4-Methyl-umbelliferyl phosphate) is cycled in and out of the SPR. The conjugate enzyme catalyzes the hydrolysis of this substrate into a fluorescent product (4-Methyl-umbelliferone) the fluorescence of which is measured at $450 \mathrm{~nm}$. The intensity of the fluorescence is proportional to the concentration of antigen present in the sample. At the end of the assay, results are automatically calculated by the instrument in relation to the calibration curve stored in memory and then printed out.

The results from both ELISA and VIDAS ${ }^{\circ}$ vWF methods were similar. For the sake of simplicity, the results derived from ELISA are presented in this study.

\section{Statistical analysis}

Parametric data for $\mathrm{vWF}$ were reported as the calculated mean \pm standard deviation.

One-way analysis of variance (ANOVA, F-test) was implemented for the analysis of the correlation of vWF follicular fluid levels and the quality of the oocytes retrieved as well as the quality of the embryos developed following fertilization of these oocytes.

Pearson parametric and Spearman non-parametric correlation tests were used to investigate the relation between follicular fluid volume and the vWF 
Table 1. Oocytes retrieved, embryos Day 1 (Zygotes), fertilization rate and mean von Willebrand factor (vWF) follicular fluid concentration per patient

\begin{tabular}{|c|c|c|c|c|}
\hline PATIENT & OOCYTES RETRIEVED & $\begin{array}{c}\text { EMBRYOS DAY1 } \\
\text { (ZYGOTES) }\end{array}$ & FERTILIZATION RATE \% & VWF MIU/I \\
\hline 1 & 6 & 4 & 66.7 & 18.96 \\
\hline 2 & 5 & 3 & 60.0 & 23.82 \\
\hline 3 & 9 & 6 & 66.7 & 22.53 \\
\hline 4 & 8 & 7 & 87.5 & 20.75 \\
\hline 5 & 10 & 7 & 70.0 & 23.7 \\
\hline 6 & 8 & 6 & 75.0 & 26.63 \\
\hline 7 & 6 & 4 & 66.7 & 24.5 \\
\hline 8 & 4 & 3 & 75.0 & 18.39 \\
\hline 9 & 5 & 5 & 100.0 & 14.92 \\
\hline 10 & 6 & 4 & 66.7 & 24.53 \\
\hline 11 & 6 & 4 & 66.7 & 39.55 \\
\hline 12 & 4 & 3 & 75.0 & 23.36 \\
\hline 13 & 15 & 14 & 93.3 & 20.8 \\
\hline 14 & 6 & 5 & 83.3 & 17.13 \\
\hline 15 & 5 & 4 & 80.0 & 18.82 \\
\hline 16 & 3 & 2 & 66.7 & 11.4 \\
\hline 17 & 2 & 2 & 100.0 & 23.15 \\
\hline 18 & 10 & 8 & 80.0 & 27.9 \\
\hline 19 & 8 & 7 & 87.5 & 17.11 \\
\hline 20 & 15 & 7 & 46.7 & 64.42 \\
\hline 21 & 9 & 5 & 55.6 & 21.44 \\
\hline 22 & 13 & 12 & 92.3 & 18.86 \\
\hline 23 & 10 & 8 & 80.0 & 32.34 \\
\hline 24 & 24 & 19 & 79.2 & 24.63 \\
\hline 25 & 7 & 7 & 100.0 & 14.24 \\
\hline
\end{tabular}

follicular fluid levels as well as vWF follicular fluid levels and fertilization rate.

\section{Results}

In Table 1, oocytes retrieved, embryos Day 1 (Zygotes), fertilization rate and mean vWF follicular fluid concentration per patient undergoing oocyte retrieval are presented. In total, 204 oocytes were collected from 25 women participating in the study and 156 out of them were fertilized resulting in Day 1 (Zygote) embryos. Interestingly, vWF was detectable in the follicular fluid of all follicles aspirated.

One-way analysis of variance (ANOVA, F-test) results revealed a correlation of vWF concentration and oocyte quality ( $\mathrm{p}$-value $=0.002$ ). In fact, low levels of vWF are correlated with better oocyte quality. Post-hoc analysis in pairs revealed statistical significant difference in mean VWF concentration 


\section{vWF vs oocyte quality (mean \pm SEM)}
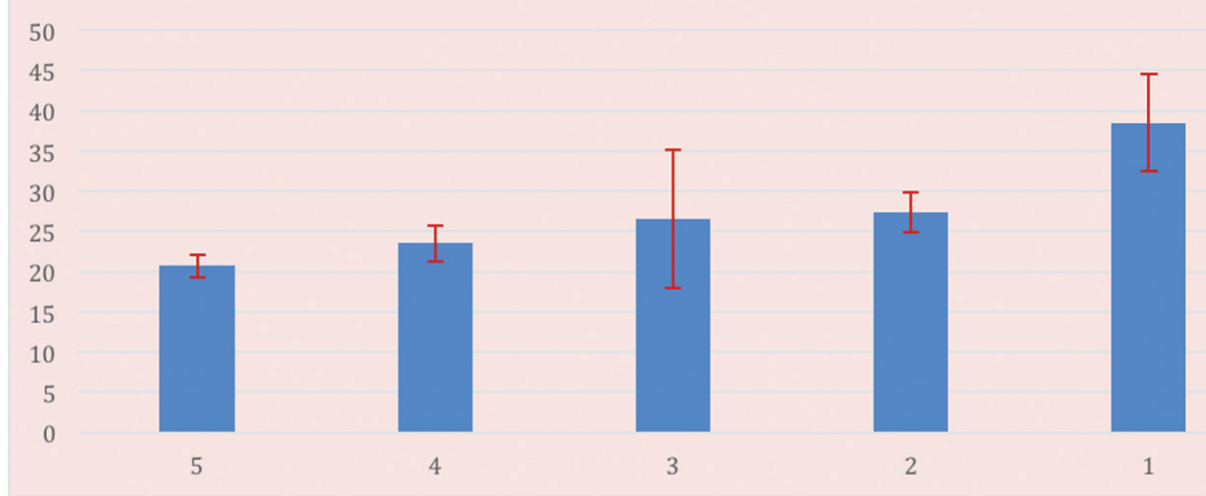

Figure 1. Correlation of von Willebrand factor follicular fluid concentration and oocyte quality.

One-way analysis of variance (ANOVA, F-test) suggested a correlation of von Willebrand Factor (vWF) follicular fluid concentration and oocyte quality ( $p$-value $=0.002$ ). In fact low levels of vWF are correlated with better oocyte quality. Post-hoc analysis in pairs revealed statistical significant difference in mean vWF concentration between oocyte quality 5 and 1 (pvalue $=0.004)$ and between oocyte quality 4 and $1(p$-value $=0.015)$

between oocyte quality 5 and $1(\mathrm{p}$-value $=0.004)$ and between oocyte quality 4 and 1 ( $p$-value $=0.015$ ) (Table 2, Figure 1).

With regards to embryo quality, one-way analysis of variance (ANOVA, F-test) suggested a correlation of $v W F$ concentration and embryo quality ( $p$-value $=0.002$ ). In fact, low levels of vWF were correlated with better embryo quality. Post-hoc analysis in pairs revealed statistical significant difference in mean $\mathrm{vWF}$ concentration between embryo quality $3+$ and 2 (pvalue $=0.004$ ) and embryo quality 3 and 2 (p-value $=0.015$ ). Having excluded cases of the oocytes not having being fertilized ( 48 cases) and cases of oocytes not having cleaved ( 2 cases), post-hoc analysis in pairs revealed statistical significant difference in mean vWF concentration between embryo quality $3+$ and $2+(p$-value $=0.034)$ and embryo quality 3 and $2+(p$-value $=0.025)($ Table 3 , Figure 2$)$.

Table 2. Correlation of von Willebrand factor follicular fluid concentration and oocyte quality.

\begin{tabular}{cccccc}
\hline & NO & OOCYTE QUALITY & MEAN VWF & STD. DEVIATION & SEM \\
& 59 & 5 & 20.68 & 10.64 & 1.38 \\
& 82 & 4 & 23.47 & 19.85 & 2.19 \\
& 4 & 3 & 26.55 & 17.24 & 8.62 \\
& 23 & 2 & 27.35 & 11.99 & 2.50 \\
\hline Total & 1 & 38.47 & 35.71 & 6.03 \\
\hline
\end{tabular}

One-way analysis of variance (ANOVA, F-test) suggested a correlation of von Willebrand Factor (VWF) follicular fluid concentration and oocyte quality ( $\mathrm{p}$-value $=0.002$ ). In fact, low levels of VWF are correlated with better oocyte quality. Post-hoc analysis in pairs revealed statistical significant difference in mean VWF concentration between oocyte quality 5 and $1(p$-value $=0.004)$ and between oocyte quality 4 and $1(p$-value $=0.015)$. 


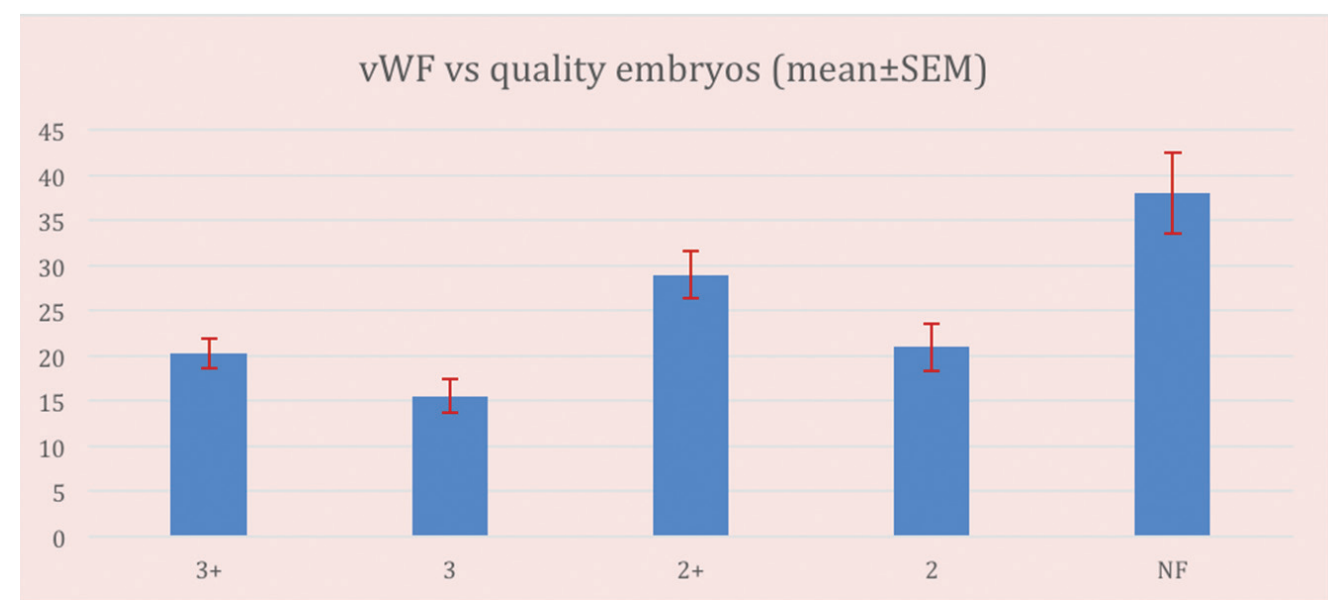

Figure 2. Correlation of von Willebrand factor follicular fluid concentration and embryo quality.

One-way ANOVA regression analysis (F-test) suggested a correlation of von Willebrand Factor (VWF) follicular fluid concentration and embryo quality ( $p$-value $=0.002$ ). In fact, low levels of vWF were correlated with better embryo quality. Post-hoc analysis in pairs revealed statistical significant difference in mean vWF concentration between embryo quality $3+$ and 2 ( $p$-value $=0.004$ ) and embryo quality 3 and 2 (p-value $=0.015$ ). Having excluded cases of the oocytes not having been fertilized ((48 cases) and cases of oocytes not having cleaved (2 cases), post-hoc analysis in pairs revealed statistical significant difference in mean vWF concentration between embryo quality $3+$ and $2+(p$-value $=0.034)$ and embryo quality 3 and $2+(p$-value $=0.025)$

NF: No Fertilization

No correlation between follicular fluid volume and vWF follicular fluid levels was found (Pearson correlation $r=-0.05$, $p$-value $=0.476$ ). This was also the fact using Spearman's correlation (Rho $=0.065$, p-value 0.355) - data not shown.
However, a negative correlation between vWF follicular fluid levels and fertilization rate was found (Pearson correlation $r=-0.531$, p-value 0.006 and Spearman's Rho $=-0.446$, p-value 0.026 ) - data not shown.

Table 3. Correlation of von Willebrand factor follicular fluid concentration and embryo quality.

\begin{tabular}{|c|c|c|c|c|c|}
\hline & NO & EMBRYO QUALITY & MEAN VWF & STD. DEVIATION & SEM \\
\hline & 95 & $3+$ & 20.27 & 15.81 & 1.62 \\
\hline & 18 & 3 & 15.48 & 7.89 & 1.86 \\
\hline & 38 & $2+$ & 28.93 & 16.26 & 2.63 \\
\hline & 3 & 2 & 20.93 & 4.47 & 2.58 \\
\hline No Fertilization & 48 & & 37.96 & 31.18 & 4.45 \\
\hline Total embryos cleaved & 154 & & 25.75 & 21.52 & 1.51 \\
\hline
\end{tabular}

One-way analysis of variance (ANOVA, F-test) suggested a correlation of von Willebrand Factor (vWF) follicular fluid concentration and embryo quality ( $p$-value $=0.002$ ). In fact, low levels of vWF were correlated with better embryo quality. Post-hoc analysis in pairs revealed statistical significant difference in mean vWF concentration between embryo quality $3+$ and $2(p$-value $=0.004)$ and embryo quality 3 and 2 (p-value $=0.015$ ). Having excluded cases of the oocytes not having been fertilized ( 48 cases) and cases of oocytes not having cleaved $(2$ cases), post-hoc analysis in pairs revealed statistical significant difference in mean vWF concentration between embryo quality $3+$ and $2+$ $(p$-value $=0.034)$ and embryo quality 3 and $2+(p$-value $=0.025)$. 


\section{Discussion}

In this study, we confirmed the presence of vWF in the follicular fluid of women undergoing IVF treatment and we found that the quality of oocytes retrieved was inversely correlated with follicular fluid vWF levels.

In fact, the higher the level of vWF in the follicular fluid the less the possibility to retrieve good quality oocytes. Actually, better quality oocytes were more likely retrieved from follicles with the lower mean vWF follicular fluid levels.

Our study also indicated an association between the levels of vWF in the follicular fluid and the quality of embryos developed from fertilization of oocytes retrieved from the corresponding follicles. In fact, good quality embryos more likely derived from follicles with the lower mean vWF follicular fluid levels.

Our findings suggest that low vWF follicular fluid levels are associated with a favorable outcome of IVF treatment in terms of oocyte as well as embryo quality.

A possible correlation between the $\mathrm{vWF}$ follicular fluid levels and follicle size reflected by the volume of the follicular fluid which was aspirated, could not be established.

To our knowledge, this is the first report of vWF detection in follicular fluid. The finding contrasts previously published data, where vWF was undetectable ${ }^{1}$. To support our findings, we have analyzed the samples with two different methods. We first measured them using an enzyme-linked immunosorbent assay (Elisa) and we further analyzed them by the VIDAS ${ }^{\circledR}$ vWF method. The latter is an automated quantitative test for the measurement of vWF in human plasma, using the ELFA technique (enzyme-linked fluorescent assay). Results were similar in both assays.

Von Willebrand factor is implicated in two essential functions of hemostasis: it mediates the adhesion of platelets to subendothelial connective tissue and it binds blood clotting factor VIII ${ }^{6}$. FVIII was not detected in follicular fluid in a previous study ${ }^{1}$. If this is the case, vWF may act through modulation of platelet adhesion. In a previous study it was shown that platelet function is periodically altered during the ovarian cycle due to the influence of progesterone and estrogen on vWF concentrations in blood ${ }^{12}$. Another retrospective study showed a consistent increase in plasma vWF levels in patients developing ovarian hyperstimulation syndrome, which lasted after embryo transfer even to the late luteal phase. Thus, plasma changes in vWF were correlated with a clinical condition characterized by increased capillary permeability which drives all subsequent morbidity ${ }^{13}$. There are changes in the localization of vWF in the follicular microvasculature in bovine ovarian follicles during atresia. The vWF-positive cells were counted in four different regions of a follicle from the apical to the basal side. In all types of follicles, immunoreactions for vWF were observed in the endothelial cells of capillaries as well as veins and arteries in the theca interna and externa. In the theca interna, the vWF-positive area was significantly greater in advanced and late atretic follicles than in healthy and early atretic follicles at all regions except for the apical region of advanced atretic follicles. The increase of vWF expression in the endothelial cells occurs before morphological changes of microvasculature appear in the atretic follicles ${ }^{14}$.

These studies suggest the presence of vWF in follicular fluid which was confirmed in the present study. The endothelial cells and microvasculature are the most likely candidates for the production of vWF in the follicular environment. The relation between oocyte and embryo quality and the concentration of vWF in follicular fluid is in accordance with recent findings that showed that the density of vWF-positive capillary vessels in the theca interna significantly increased as atresia progressed in swamp buffalo $(\mathrm{SB})$, whereas the density significantly decreased in 
late atretic follicles compared with advanced ones in Hollstein-Friesian cows (HF) and that the area of vWF-positive capillary vessels in the theca interna significantly increased as atresia progressed in both $\mathrm{SB}$ and $\mathrm{HF}^{14}$.

As noted above, plasma changes in vWF were correlated with the clinical condition of hyperstimulated patients, since the rise of capillary permeability is the central event in all subsequent morbidity ${ }^{13}$. This could be related locally in the follicle with changes in the steroid environment. It has been demonstrated that oocytes require a specific intrafollicular steroid environment for the inductive signals of meiotic resumption and the completion of the full maturational process ${ }^{15,16}$. Steroids exert a significant influence on the synthesis of cytoplasmic factors that induce normal decondensation of the sperm head and formation of the male pronucleus ${ }^{17}$. Similarly, results showing that ovarian steroid production is not essential for the resumption of meiosis but participates in cytoplasmic maturation of the oocyte have been reported ${ }^{18}$.

In the past, many investigators have focused on the implementation of non-invasive tools in the assessment of oocyte and/or embryo quality, such as glucose uptake, pyruvate concentration and plateletactivating factor (PAF) levels ${ }^{19}$. Such methods have not been applied in the clinical setting so far, mainly due to the lack of strong evidence of their clinical significance or due to practical difficulties associated with their application. The need for a "prognostic" factor is more evident in conventional IVF in which oocyte maturity is assessed indirectly, while in ICSI, the morphological structure of denuded oocytes can be assessed in more detail ${ }^{20}$. From our data, it seems that vWF follicular levels could stand as such a factor, which could contribute in the prediction and evaluation of both oocyte and embryo quality.

Future studies aiming to elucidate whether a relationship between the levels of vWF in plasma and follicular fluid exists, could potentially shift our attention from follicular fluid to plasma for the determination of this "promising" parameter.

\section{References}

1. Gentry PA, Plante L, Schroeder MOB, LaMarre J, Young JE, Dodds WG. Human ovarian follicular fluid has functional systems for the generation and modulation of thrombin. Fertil Steril 2000; 74:848-854.

2. Gosden RG, Hunter RH, Telfer E, Torrance C, Brown N. Physiological factors underlying the formation of ovarian follicular fluid. J Reprod Fertil 1988;82(2):813-25.

3. Nagy B, Pulay T, Szarka G, Csomor S. The serum protein content of human follicular fluid and its correlation with the maturity of oocytes. Acta Physiol Hung 1989;73(1):71-5.

4. Shalgi R, Kraicer P, Rimon A, Pinto M, Soferman N. Proteins of human follicular fluid: the bloodfollicle barrier. Fertil Steril 1973;24(6):429-34.

5. Lehwald-Van Voorst N.A.M. Protein profile of bovine follicular fluid and the synthesis of fibronectin by bovine granulosa cells. MSc thesis 1993, University of Guelph.

6. Sadler JE. Biochemistry and genetics of von Willebrand factor. Annu Rev Biochem 1998;67: 395-424.

7. Wagner DD, Olmsted JB, Marder VJ. Immunolocalization of von Willebrand protein in Weibel-Palade bodies of human endothelial cells. J Cell Biol. 1982;95(1):355-60.

8. Sporn LA, Marder VJ, Wagner DD. Inducible secretion of large, biologically potent von Willebrand factor multimers. Cell. 1986;46(2):185-90.

9. Isobe N, Kawai H, Yoshimura Y, Nakao T. Changes in the localization of immunoreactive von Willebrand factor in microvascular network of bovine ovarian follicles during atresia. Animal Science J 2001;72(6):473-82.

10. Loutradis D, Drakakis P, Kallianidis K, Bletsa R, 
Milingos S, Makris N, et al. The effect of the duration of GnRH-agonist down regulation before ovarian stimulation on the biological and clinical outcome after intracytoplasmic sperm injection. Eur J Obstet Gynecol Reprod Biol 1998;80(2):251-5.

11. Loutradis D, Elsheikh A, Kallianidis K, Drakakis P, Stefanidis K, Milingos S, et al. Results of controlled ovarian stimulation for ART in poor responders according to the short protocol using different gonadotrophins combinations. Arch Gynecol Obstet. 2004;270(4):223-6.

12. Feuring M, Christ M, Roell A, Schueller P, Losel R, Dempfle CE, et al. Alterations in platelet function during the ovarian cycle. Blood Coagul Fibrinolysis 2002;13(5):443-7.

13. Todorow S, Schricker ST, Siebzehnruebl ER, Neidhardt B, Wildt L, Lang N. Von Willebrand factor: an endothelial marker to monitor in-vitro fertilization patients with ovarian hyperstimulation syndrome. Hum Reprod 1993;8(12):2039-46.

14. Feranil JB, Isobe N, Nakao N. Immunolocalization of von Willebrand factor and vascular endothelial growth factor during follicular atresia in the swamp buffalo ovary. J Reprod Dev, 51(4):419-26, 2005

15. Moor RM, Polge C, Willandsen SM. Effect of follicular steroids on the maturation and fertilization of mammalian oocytes. J Embryol Exp Morphol 1980;56:319-24.

16. Ainsworth L. Tsang BK, Downey BR, Marcus GJ,
Armstrong DI. Interrelationships between follicular fluid steroid levels, gonadotropic stimuli, and oocyte maturation during preovulatory development of porcine follicles. Biol Reprod 1980;23:261-6.

17. Thibault C, Gerard M, Menezo Y. Acquisition par l' oocyte de lapine et de veau du facteur de decondensation du nuyau du spermatozoide fecondant (MPGF). Ann Biol Anim Biochim Biophys 1975;15:705-9.

18. Yoshimura Y, Hosoi Y, Bongiovani AM, Santulli R, Atlas SJ, Wallach EE. Are ovarian steroids required for ovum maturation and fertilization? Effects of cyanoketone on the in vitro perfused rabbit ovary. Endocrinology 1987;120:2555-61.

19. Loutradis D, Drakakis P, Kallianidis K, Sofikitis N, Kallipolitis G, Milingos S, Makris N, Michalas $\mathrm{S}$. Biological factors in culture media affecting in vitro fertilization, preimplantation embryo development and fertilization. Ann N Y Acad Sci 2000;900: 325-35

20. Loutradis D, Drakakis P, Kallianidis K, Milingos S, Dendrinos S, Michalas S. Oocyte morphology correlates with embryo quality and pregnancy rate after intracytoplasmic sperm injection. Fertil Steril 72:240-244, 1999.

Received 25-02-21

Revised 02-03-21

Accepted 08-03-21 